\title{
A SUFFICIENT CONDITION FOR A REGULAR MATRIX TO SUM A BOUNDED DIVERGENT SEQUENCE
}

\section{A. MARY TROPPER}

If a matrix $A$ transforms a sequence $\left\{z_{n}\right\}$ into the sequence $\left\{\sigma_{n}\right\}$, i.e., if $\sigma_{n}=\sum_{k-1}^{\infty} a_{n, k} z_{k}$, and if $\sigma_{n} \rightarrow z$ as $n \rightarrow \infty$ whenever $z_{n} \rightarrow z, A$ is said to be regular. The well known necessary and sufficient conditions for $A$ to be regular are ${ }^{1}$

(a) $\sum_{\mathbf{k}-1}^{\infty}\left|a_{n, k}\right|<M$ for every positive integer $n>n_{0}$,

(b) $\lim _{n \rightarrow \infty} a_{n, k}=0$ for every fixed $k$,

(c) $\sum_{k=1}^{\infty} a_{n, k} \equiv A_{n} \rightarrow 1$ as $n \rightarrow \infty$.

It is known ${ }^{2}$ that if a regular matrix sums a bounded divergent sequence, then it also sums some unbounded sequence. The converse is, however, false. ${ }^{8}$ It is consequently of interest to find sufficient conditions for a regular matrix to sum a bounded divergent sequence. Many authors have considered summability of bounded sequences." R. P. Agnew has given a simple sufficient condition that a regular matrix shall sum a bounded divergent sequence. He has proved that if $A$ is a regular matrix such that $\lim _{n, k \rightarrow \infty} a_{n, k}=0$, then some divergent sequences of 0 's and 1's are summable- $A$. There are, however, very many simple regular matrices which do not satisfy this condition, but which are known to sum a bounded divergent sequence. For example, the matrix $A$ obtained by replacing every third row of the Cesàro matrix $(C, 1)$ by the corresponding row of the unit matrix, given by

$$
\begin{array}{rlrl}
a_{3 n-2, k} & =\frac{1}{3 n-2} \quad(k \leqq 3 n-2), & a_{3 n-1, k}=\frac{1}{3 n-1} \quad(k \leqq 3 n-1), \\
a_{3 n, k} & =\delta_{3 n, k}, & a_{n, k}=0 \quad(k>n) & (n, k=1,2, \cdots),
\end{array}
$$

sums the sequence $\{0,2,1,0,2,1,0, \cdots\}$ to the limit 1 . This matrix does, however, satisfy the conditions which will be given in Theorem II.

I first show that I need consider only normal matrices, i.e., lower-

Received by the editors November 10, 1952 and, in revised form, January 16, 1953. ${ }^{1}$ See R. G. Cooke [1, pp. 64-65].

2 Stated without proof by S. Mazur and W. Orlicz [2]; a proof is given by V. M. Darevsky [3]. See also J. D. Hill [4]; A. Wilansky [5]; K. Zeller [6].

${ }^{3}$ See R. G. Cooke [1, p. 178, Examples 7, no. 10].

4 See, e.g., G. G. Lorentz $[7 ; 8] ;$ R. P. Agnew [9]; A. Wilansky [10; 11].

6 R. P. Agnew [12, pp. 128-132]; this is a special case of G. G. Lorentz [7, p. 181, Theorem 8 and footnote]. 
semi-matrices with no zero element in the leading diagonal. A normal matrix has a unique right-hand reciprocal which is also normal, and which is also a left-hand reciprocal..$^{\circ}$ If a matrix $B$ is such that $\|B\|$ $=\sup _{n} \sum_{k}\left|b_{n, k}\right|=\infty$, then, by a method now classical, we can construct a null sequence whose $B$-transform is unbounded. It is not, in general, possible to construct a null sequence whose $B$-transform is bounded and divergent. This can be done, however, if $B$ is normal and its columns form null sequences. This is the main result of this paper and its interest lies in its sufficiency that $B^{-1}=A$ shall sum a bounded divergent sequence.

The following theorem is due to A. Brudno. ${ }^{7}$ Brudno's proof, however, is somewhat complicated, and I give here a simpler proof.

THEOREM I. If $A$ is a general (square) regular matrix, there exists $a$ normal regular matrix $A^{*}$, such that $A$ and $A^{*}$ are mutually consistent ${ }^{8}$ for bounded sequences.

Proof. Let $\left\{\epsilon_{n}\right\}$ be any null sequence with $\epsilon_{n}>0$ for each $n$. Since $A$ is regular, by (a) we can choose a monotonic increasing sequence of positive integers $\left\{p_{n}\right\}(n=1,2, \cdots)$ such that

$$
\sum_{k=p_{n}+1}^{\infty}\left|a_{n, k}\right|<\epsilon_{n} \quad \text { for every } n .
$$

Let the matrix $A^{*}$ be given by

$$
\begin{array}{ll}
a_{n, k}^{*}=a_{1, k} & \left(1 \leqq k<n<p_{1}\right), \\
a_{n, n}^{*}=\left\{\begin{array}{ll}
a_{1, n} & \left(a_{1, n} \neq 0\right) \\
1 / n & \left(a_{1, n}=0\right)
\end{array}\right\} & \left(n<p_{1}\right), \\
a_{n, k}^{*}=a_{l, k} & \left(p_{l} \leqq n<p_{l+1}, l \geqq 1,1 \leqq k<n\right), \\
a_{n, n}^{*}=\left\{\begin{array}{ll}
a_{l, n} & \left(a_{l, n} \neq 0\right) \\
1 / n & \left(a_{l, n}=0\right)
\end{array}\right\} \quad\left(p_{l} \leqq n<p_{l+1}, l \geqq 1\right), \\
a_{n, k}^{*}=0 & (k>n) .
\end{array}
$$

Let $\sigma_{n}=A\left(z_{n}\right)=\sum_{k=1}^{\infty} a_{n, k} z_{k}, \rho_{n}=A^{*}\left(z_{n}\right)=\sum_{k=1}^{n} a_{n, k}^{*} z_{k}$. If $p_{l} \leqq n<p_{l+1}$, $\sigma_{l}-\rho_{n}=\sum_{k=n+1}^{\infty} a_{l, k} z_{k}+\left(a_{l, n}-a_{n, n}^{*}\right) z_{n}$. Hence, if $\left\{z_{n}\right\}$ is a bounded sequence for which $\left|z_{n}\right| \leqq M$ for every $n$,

- R. G. Cooke [1, pp. 19, 22].

7 A. Brudno [13].

- I.e., $A^{*}$ sums, to the same limit, every bounded sequence which is summable- $A$ and vice versa. 


$$
\begin{aligned}
\left|\sigma_{l}-\rho_{n}\right| & \leqq M \sum_{k=p_{l+1}}^{\infty}\left|a_{l, k}\right|+\frac{M}{n} \\
& <M\left(\epsilon_{l}+\frac{1}{n}\right) \rightarrow 0 \quad \text { as } l \rightarrow \infty
\end{aligned}
$$

since $n$ and $l$ tend to $\infty$ together.

Thus $A\left(z_{n}\right)$ and $A^{*}\left(z_{n}\right)$ either both converge to the same limit, or neither converges, and $A^{*}$ is normal.

I now prove the main theorems.

THEOREM II. In order that the regular normal matrix $A$ shall sum a bounded divergent sequence it is sufficient that its unique two-sided reciprocal $B$ shall not be regular, and that all the columns of $B$ shall form bounded sequences.

THEOREM III. In order that the regular normal matrix $A$ shall sum $a$ bounded divergent sequence it is sufficient that

(a) its unique reciprocal $B$ shall not be regular, and

(b) there exists a normal matrix $Q$ with $\|Q\|<\infty$, whose columns are all null sequences, such that the matrix $C=B Q$ has bounded columns and $\|C\|=\infty$.

Proof of Theorem III. If $A\left(z_{n}\right)=\sigma_{n}$, then

$$
B\left(\sigma_{n}\right)=B\left[A\left(z_{n}\right)\right]=(B A)\left(z_{n}\right)=\left(z_{n}\right),
$$

the alteration in the order of summation being justified, since only finite sums are involved.

If $B$ is regular, $\left\{z_{n}\right\}$ converges whenever $\left\{\sigma_{n}\right\}$ converges, so that $A$ sums only convergent sequences. If $B$ is not regular, there exists a convergent sequence $\left\{\sigma_{n}\right\}$ such that $\left\{z_{n}\right\}$ is divergent. Thus, in order that $A$ shall be stronger than convergence it is necessary and sufficient that $B$ shall not be regular.

Since $B$ and $Q$ are normal, $C=B Q$ is also normal, and hence

$$
A C=A(B Q)=(A B) Q=Q,
$$

so that, assuming condition (b), $A$ transforms each column of $C$ into a null sequence. Since $A$ is regular, it follows that each column of $C$ is either a divergent or a null sequence. If at least one column of $C$ is divergent, the result is proved. There remains to be considered only the case in which all the columns of $C$ form null sequences. Thus $c_{n, k} \rightarrow 0$ as $n \rightarrow \infty$ for every fixed $k$, and if $M_{n}=\sum_{k=1}^{n}\left|c_{n, k}\right|$, the sequence $\left\{M_{n}\right\}$ is unbounded, by hypothesis, and therefore has a subsequence which tends to infinity. 
If $Z=\mathrm{re}^{i \theta}$, let $\operatorname{sgn} Z=e^{-i \theta}(Z \neq 0), \operatorname{sgn} 0=0$.

Choose a positive integer $n_{1}$ such that $M_{n_{1}}>M_{n}$ for all $n<n_{1}$. Put

$$
x_{k}=\frac{\operatorname{sgn}\left(c_{n_{1}, k}\right)}{M_{n_{1}}}
$$

If $C\left(x_{n}\right)=y_{n}$,

$$
y_{n_{1}}=\sum_{k=1}^{n_{1}} c_{n_{1}, k} x_{k}=\frac{1}{M_{n_{1}}} \sum_{k=1}^{n_{1}}\left|c_{n_{1}, k}\right|=1 .
$$

Let $\epsilon>0$ be fixed and arbitrarily small. We can choose $n_{2}>n_{1}$ such that

$$
\sum_{k=1}^{n_{1}}\left|c_{n, k}\right|<\frac{1}{2} \epsilon \quad \text { for every } n \geqq n_{2}
$$

and

$$
M_{n_{2}}>M_{n} \quad \text { for every } n<n_{2} .
$$

Put

$$
x_{k}=-\frac{\operatorname{sgn}\left(c_{n_{2}, k}\right)}{M_{n_{2}}} \quad\left(n_{1}<k \leqq n_{2}\right) .
$$

Then

$$
\begin{aligned}
y_{n_{2}} & =\sum_{k=1}^{n_{1}} c_{n_{2}, k} x_{k}-\sum_{k=n_{1}+1}^{n_{2}} \frac{c_{n_{2}, k} \operatorname{sgn}\left(c_{n_{2}, k}\right)}{M_{n_{2}}} \\
& =\frac{1}{M_{n_{1}}} \sum_{k=1}^{n_{1}} c_{n_{2}, k} \operatorname{sgn}\left(c_{n_{1}, k}\right)-\frac{1}{M_{n}} \sum_{k=n_{1}+1}^{n_{2}}\left|c_{n_{2}, k}\right| .
\end{aligned}
$$

We now choose $n_{3}>n_{2}$ such that

$$
\sum_{k=n_{1}+1}^{n_{2}}\left|c_{n, k}\right|<\frac{1}{2^{2}} \epsilon \quad \text { for every } n \geqq n_{3},
$$

and

$$
M_{n_{3}}>M_{n} \quad \text { for every } n<n_{3}
$$

Put

$$
x_{k}=\frac{\operatorname{sgn}\left(c_{n_{3}, k}\right)}{M_{n_{3}}} \quad\left(n_{2}<k \leqq n_{3}\right) .
$$

Then 


$$
\begin{aligned}
y_{n_{3}}= & \frac{1}{M_{n_{1}}} \sum_{k=1}^{n_{1}} c_{n_{3}, k} \operatorname{sgn}\left(c_{n_{1}, k}\right)-\frac{1}{M_{n_{2}}} \sum_{k=n_{1}+1}^{n_{2}} c_{n_{3}, k} \operatorname{sgn}\left(c_{n_{2}, k}\right) \\
& +\frac{1}{M_{n_{3}}} \sum_{k=n_{2}+1}^{n_{8}}\left|c_{n_{3}, k}\right| .
\end{aligned}
$$

Continue in this way; thus

$$
x_{k}=(-1)^{p-1} \frac{\operatorname{sgn}\left(c_{n_{p}, k}\right)}{M_{n_{p}}} \quad\left(n_{p-1}<k \leqq n_{p}\right) .
$$

For any integer $p$,

$$
\begin{aligned}
1-\frac{1}{M_{n_{p}}} \sum_{k=n_{p-1}+1}^{n_{p}}\left|c_{n_{p}, k}\right| & =\frac{1}{M_{n_{p}}}\left\{\sum_{k=1}^{n_{1}}\left|c_{n_{p}, k}\right|+\sum_{k=n_{1}+1}^{n_{2}}\left|c_{n_{p}, k}\right|+\cdots\right. \\
& \left.+\sum_{k=n_{p-2}+1}^{n_{p-1}}\left|c_{n_{p}, k}\right|\right\} \\
& <\frac{1}{M_{n_{p}}}\left\{\frac{1}{2} \epsilon+\frac{1}{2^{2}} \epsilon+\cdots+\frac{1}{2^{p-1}} \epsilon\right\} \\
& <\frac{\epsilon}{M_{n_{p}}} \rightarrow 0 \quad \text { as } p \rightarrow \infty,
\end{aligned}
$$

and is arbitrarily small for $p=1,2,3, \ldots$. If $p$ is odd,

$$
\begin{aligned}
& \left|y_{n_{p}}-\frac{1}{M_{n_{p}}} \sum_{k=n_{p-1}+1}^{n_{p}}\right| c_{n_{p}, k}|| \\
& <\frac{1}{M_{n_{1}}} \sum_{k=1}^{n_{1}}\left|c_{n_{p}, k}\right|+\frac{1}{M_{n_{2}}} \sum_{k=n_{1}+1}^{n_{2}}\left|c_{n_{p, k}}\right|+\cdots+\frac{1}{M_{n_{p-1}}} \sum_{k=n_{p-1}+1}^{n_{p-1}}\left|c_{n_{p, k}}\right| \\
& <\frac{1}{M_{n_{1}}} \cdot \frac{1}{2} \epsilon+\frac{1}{M_{n_{2}}} \cdot \frac{1}{2^{2}} \epsilon+\cdots+\frac{1}{M_{n_{p-1}}} \cdot \frac{1}{2^{p-1}} \epsilon \\
& <\frac{1}{M_{n_{1}}} \epsilon\left(\frac{1}{2}+\frac{1}{2^{2}}+\cdots+\frac{1}{2^{p-1}}\right) \\
& <\frac{\epsilon}{M_{n_{1}}}
\end{aligned}
$$

which is arbitrarily small. The last two inequalities together show that

$$
y_{n_{p}}-1 \text { can be made arbitrarily small when } p \text { is odd. }
$$

Similarly $y_{n_{p}}+1$ can be made arbitrarily small when $p$ is even. Thus the sequence $\left\{y_{n}\right\}$ is divergent. Moreover, if $n_{q}<n \leqq n_{q+1}$, 


$$
\begin{aligned}
\left|y_{n}\right| \leqq & \frac{1}{M_{n_{1}}} \sum_{k=1}^{n_{1}}\left|c_{n, k}\right|+\frac{1}{M_{n_{2}}} \sum_{k=n_{1}+1}^{n_{z}}\left|c_{n, k}\right|+\cdots \\
& +\frac{1}{M_{n_{q}}} \sum_{k=n_{q-1}+1}^{n_{q}}\left|c_{n, k}\right|+\frac{1}{M_{n_{q+1}}} \sum_{k=n q+1}^{n}\left|c_{n, k}\right| \\
< & \frac{1}{M_{n_{1}}}\left\{\frac{1}{2} \epsilon+\frac{1}{2^{2}} \epsilon+\cdots+\frac{1}{2^{q}} \epsilon\right\}+\frac{M_{n}}{M_{n_{q+1}}} \\
& <\frac{\epsilon}{M_{n_{1}}}+1, \text { since } M_{n}<M_{n_{q+1}} .
\end{aligned}
$$

Thus $\left\{y_{n}\right\}$ is a bounded divergent sequence, and $y_{n}=C\left(x_{n}\right)$, where $\left\{x_{n}\right\}$ is a null sequence.

Hence $B\left[Q\left(x_{n}\right)\right]=(B Q)\left(x_{n}\right)=C\left(x_{n}\right)=y_{n}$. Let $Q\left(x_{n}\right)=\xi_{n}$. Now since $\|Q\|<\infty$ and $q_{n, k} \rightarrow 0$ as $n \rightarrow \infty$ for every fixed $k$, it follows ${ }^{9}$ that $Q$ transforms every null sequence into a null sequence. Thus $\left\{\xi_{n}\right\}$ is a null sequence and $B\left(\xi_{n}\right)=y_{n}$. Hence $A\left(y_{n}\right)=\xi_{n}$, and $A$ sums the bounded divergent sequence $\left\{y_{n}\right\}$ to the limit zero.

The theorem is now proved.

For $Q=I$, Theorem II follows. For, in this case, $M_{n}=\sum_{k=1}^{n}\left|b_{n, k}\right|$. It is obvious that the sequence $\left\{M_{n}\right\}$ is unbounded; for if $M_{n}<M$ for every $n, B$ would transform every convergent sequence into a bounded sequence. This would imply that all the divergent sequences which are summable- $A$ are bounded. This is impossible, as already mentioned.

Corollary. The theorem still holds if all but a finite number of the columns of $C$ form bounded sequences.

If all but the first $N$ columns are bounded, we put $x_{k}=0(k \leqq N)$. Define $\left\{M_{n}\right\}$ by the equation $M_{n}=\sum_{k=N+1}^{n}\left|c_{n, k}\right|(n>N)$, and with slight modifications the proof proceeds as before.

Examples. The matrix $A$, already quoted, obtained by modifying the $(C, 1)$ matrix, has reciprocal $B$ given by

$$
\begin{aligned}
b_{3 n, 3 n} & =1, \quad b_{3 n-1,3 n-1}=3 n-1, \quad b_{3 n-1,3 n-2}=-(3 n-2), \\
b_{3 n-2,3 n-2} & =3 n-2, \quad b_{3 n-2,3 n-3}=-1, \\
b_{3 n-2,3 n-4} & =-(3 n-4), \quad b_{n, k}=0 \text { otherwise. }
\end{aligned}
$$

$B$ is not regular, and every column of $B$ tends to zero. The conditions of Theorem II are satisfied.

\footnotetext{
' See, e.g., R. G. Cooke [1, p. 64].
} 
P. Vermes has suggested the following example of a matrix which satisfies the conditions of Theorem III.

Let $U$ be the matrix for which $u_{n+1, n}=1, u_{n, k}=0$ otherwise. Take $A=2^{-p}(I+U)^{p}, p$ being a positive integer $\geqq 2$; then $A$ is regular, and sums the sequence $\{1,0,1,0,1,0, \cdots\}$ to $1 / 2 . B=2^{p}(I+U)^{-p}$ is not regular and its columns are not bounded. Take $Q=(I+U)^{p-1}$; then $\|Q\|=2^{p-1}$ and $Q$ has zero column limits. Thus $C=B Q$ $=2^{p}(I+U)^{-1}$, which has bounded columns, and $\|C\|=\infty$.

I am unable to prove that the conditions of Theorem III are also necessary.

In conclusion I should like to thank Dr. R. G. Cooke and Dr. P. Vermes, who read the manuscript, for their helpful suggestions.

\section{REFERENCES}

1. R. G. Cooke, Infinite matrices and sequence spaces, Macmillan, 1950.

2. S. Mazur and W. Orlicz, Sur les méthodes linéaires de sommation, C. R. Acad. Sci. Paris vol. 196 (1933) pp. 32-34.

3. V. M. Darevsky, On intrinsically perfect methods of summation, Bull. Acad. Sci. URSS. Sér. Math. vol. 10 (1946) pp. 97-104.

4. J. D. Hill, Some properties of summability, Bull. Amer. Math. Soc. vol. 50 (1944) pp. 227-230.

5. A. Wilansky, A necessary and sufficient condition that a summability method be stronger than convergence, Bull. Amer. Math. Soc. vol. 55 (1949) pp. 914-916.

6. K. Zeller, Allgemeine Eigenschaften von Limitierungsverfahren, Math. Zeit. vol. 53 (1951) pp. 463-487.

7. G. G. Lorentz, A contribution to the theory of divergent sequences, Acta Math. vol. 80 (1948) pp. 167-190.

8. - Direct theorems on methods of summability, Canadian Journal of Mathematics vol. 1 (1949) pp. 305-319 and vol. 2 (1951) pp. 236-256.

9. R. P. Agnew, Convergence fields of methods of summability, Ann. of Math. (2) vol. 46 (1945) pp. 93-101.

10. A. Wilansky, An application of Banach linear functionals to summability, Trans. Amer. Math. Soc. vol. 67 (1949) pp. 59-68.

11. - Norms of matrix type for the spaces of convergent and bounded sequences, Proceedings of the American Mathematical Society vol. 2 (1951) pp. 738-741.

12. R. P. Agnew, A simple sufficient condition that a method of summability be stronger than convergence, Bull. Amer. Math. Soc. vol. 52 (1946) pp. 128-132.

13. A. Brudno, Summation of bounded sequences by matrices, Rec. Math. (Mat. Sbornik) N.S. vol. 16 (1945) pp. 191-247.

Queen Mary College, University of London 